\title{
Self-Directed Learning During the COVID-19 Pandemic: Perspectives of South African Final-Year Health Professions Students
}

\author{
Veena S Singaram (iD) \\ Kimesh L Naidoo ${ }^{2}$ \\ Shenuka Singh iD ${ }^{3}$ \\ 'Clinical and Professional Practice, School \\ of Clinical Medicine, College of Health \\ Sciences, University of KwaZulu-Natal, \\ Durban, South Africa; ${ }^{2}$ Paediatrics and \\ Child Health, School of Clinical Medicine, \\ College of Health Sciences, University of \\ KwaZulu-Natal, Durban, South Africa; \\ ${ }^{3}$ Discipline of Dentistry, School of Health \\ Sciences, College of Health Sciences, \\ University of KwaZulu-Natal, Durban, South \\ Africa
}

Purpose: Self-directed learning (SDL) has been advocated for effective training of final-year health professions students. COVID-19 challenges conventional teaching, learning, and assessment in the clinical environment. This study aimed to identify and explore enablers and barriers to SDL among final-year health professions students training during the COVID-19 pandemic.

Methods: Adopting the SWOT (strengths, weaknesses, opportunities and threats) framework, this study explored the clinical learning and training experiences of final-year health professions students during the pandemic. A survey was conducted via online platforms. Data from 155 respondents were thematically analyzed.

Results: Personal attributes such as reflection, self-determination, motivation, resilience, and positive learning behaviors and skills were identified as SDL enablers. Collaborative learning networks and online learning platforms facilitate learning needs and goals. Fear and anxiety, untrusted learning sites, uncertainty about graduation, financial issues and challenges in the learning environments were the major themes related to barriers to SDL.

Conclusion: The importance of SDL as a skill for uncertain times warrants further investigation in the training of future healthcare professionals. Inclusive planning and engagement with final-year health professions students to address identified stressors, as well as the creation of shared platforms where students are part of the decision-making processes for clinical learning and training are recommended. Responsive curricula that optimize unpredictable disruptions in clinical training are needed to equip students to diagnose their own learning needs and implement appropriate learning strategies.

Keywords: undergraduate medical students, learning environment, clinical training, medical curriculum, self-directed learning, COVID-19, health professions students

\section{Introduction}

The COVID-19 pandemic has created an uncertain and stressful environment for both faculty and students. ${ }^{1}$ This has inevitably affected teaching and learning. ${ }^{2}$ Reported challenges to teaching and learning include the lack of in-person support, lack of communication and peer interaction, and the rapid upscaling of online technologies. ${ }^{3-5}$ Health professions education is more than simply the acquisition of knowledge and skills as it also includes learning within collaborative clinical workplaces. ${ }^{6}$ Face-to-face learning within these authentic clinical environments can help build confidence, improve communication skills, and foster team building and networking. The COVID-19 pandemic potentially places barriers on many of these aspects of teaching and learning.
Correspondence: Veena S Singaram

Email singaram@ukzn.ac.za 
The application of online teaching and assessment for courses that were designed initially for face-to-face contact could be a challenge for both faculty and students. ${ }^{7}$ The need for social distancing and decreased access to patient care due to stricter infection control practices (quarantine and isolation) complicate student-centered clinical teaching and learning. ${ }^{6,8}$ Challenges with the availability of personal protective equipment (PPE) place further strains on working and learning in the clinical environment. $^{9}$

Self-directed learning (SDL), a critical component of andragogy, has been advocated as an efficient and effective skill for medical students, residents, practicing doctors, nurses, and other healthcare professionals. ${ }^{10-12}$ SDL, as defined by Knowles, is a process in which individuals take the initiative, with or without the help of others, in diagnosing their learning needs, formulating goals, identifying human and material resources for learning, choosing and implementing appropriate learning strategies, and evaluating learning outcomes. ${ }^{13}$ This SDL theory has relevance now as it did in earlier years, as it is "a longstanding skill required especially for uncertain times". ${ }^{14}$

However, little is currently documented on SDL in the context of the COVID-19 pandemic and whether this crisis has enabled or challenged this approach to learning. Mahlaba, in his recent paper outlining the reasons why SDL is important during the COVID-19 pandemic, has highlighted the need for SDL as a response to the new normal, foregrounding significant issues around the importance of self-directed medical training. ${ }^{16} \mathrm{SDL}$, under the new COVID-19 normal of remote learning and the utility of expanded digital learning platforms, enables medical students to participate in the knowledge production economy at a global level while "salvaging the academic year during the COVID-19 pandemic". ${ }^{16}$ Yet, at the same time, much of the undergraduate teaching tends to focus on the taught (expressed) curriculum. Little is known on how these students cope with the stressors (hidden curriculum) or of the skills needed to adapt to a changing environment, especially in the context of the COVID-19 pandemic. Final-year students would be most exposed to COVID19 , given that this is the exit level of the undergraduate training programmes.

Additionally, this exit year is before salaried internships, and in primarily resource-constrained environments, this comes with often suboptimal supervision. Thus, the novelty of this study lies in its focus on final-year health science students in the context of clinical training, specifically in relation to creating a picture to better understand how students cope and adapt to sudden changes in the learning environment. Such information would be valuable for curriculum development and review.

Further, health professions students are the future healthcare workforce, and they should play key roles in response to public health emergencies. Yet, in the face of the current pandemic, "they are being largely considered non-essential in clinical delivery". ${ }^{6}$ Consequently, an urgent review of the roles and responsibilities of final-year students is required. The need for and emphasis on SDL as a focused approach in health professions education cannot be undervalued. This is especially important in final-year curricula that are expected to infuse this approach across all teaching, learning, and assessment activities. Barriers to achieving SDL goals, however, have been documented and can be categorized into difficulty with personal reflection, environmental strain, competing demands, difficulty with goal generation, and problems with plan development and implementation. ${ }^{11}$ Hence, exploring the impact of the COVID-19 pandemic on SDL is both critical and opportune, as "preparing future professionals for highly dynamic settings require robust SDL skills development" ${ }^{15}$ This study aims to identify and explore both enablers and barriers to SDL in within the final-year health professional training environment during the COVID-19 pandemic.

\section{Methods Study Design}

A descriptive qualitative study using a SWOT (strengths, weaknesses, opportunities and threats) framework was conducted. A SWOT framework was adopted as it is reported as a resourceful situation analysis technique to identify strengths, weaknesses, threats and opportunities. $^{17,19}$

\section{Ethics Approval and Consent to Participate}

This study received approval from the University of KwaZulu-Natal Ethics Committee (HSSREC/00001306/ 2020). All participants informed consent included publication of anonymized responses.

\section{Setting}

South Africa (SA) has been the African country most severely affected by COVID-19. Already burdened with HIV and tuberculosis epidemics, academic hospitals in SA, 
which provide the learning environments for health professions students and provide health care to large numbers of the SA public, have had to rapidly adjust to an additional burden. Multiple health professional faculties within universities are aligned to various academic hospital training platforms. The health faculties develop the curricula and are responsible for much of the teaching and learning and assessment activities. The setting for this study is thus based within these public, academic hospitals in urban centers in SA, which reflects resource-constrained environments in many developing countries.

\section{Data Collection and Sampling}

We performed a cross-sectional qualitative survey specifically targeting final-year health professions students registered in institutions of higher education in SA during the first wave of the of the COVID-19 pandemic (MayJune 2020).

The online survey instrument used to generate the data was created using SurveyMonkey (SurveyMonkey Inc., San Mateo, California, USA, www.surveymonkey.com) http://www.surveymonkey.com) by VSS and modified by $\mathrm{KN}$ and SS. SurveyMonkey is an online platform that allows researchers to construct and disseminate selfadministered questionnaires and is suitable for open-ended questions. This survey included a combination of closeended demographic questions (sex, institution, marital status) and eight open-ended questions. The survey was piloted with four medical students for face validity before the online hyperlink was shared via the WhatsApp ${ }^{\circledR}$ social media platform. Minor modifications to the wording of questionnaires were made based on the the feedback from the pilot.

As Braun et $\mathrm{a}^{18}$ outlined, although qualitative surveys are novel, online qualitative surveys are an accepted form of collecting rich qualitative data as respondents can type their responses in their own words. Hence, qualitative surveys with open-ended questions allow participants to control how they define themselves and produce rich and complex data related to the respondents' own experiences, narratives, and practices. In the open-ended questions, participants were asked to provide insights related to their personal and professional strengths, weaknesses, opportunities and threats of clinical training during COVID-19. The survey included questions such as "List any personal strengths related to clinical training during the COVID-19 pandemic" and "List any professional strengths related to clinical training during the COVID-
19 pandemic" were included for each of the SWOT domains.

Snowball sampling was adopted for this study, as accessing participants during the pandemic was challenging due to social distancing regulations. Final-year student representatives within each of the major academic health institutions in SA were invited to share the survey with their peers via WhatsApp or any other social media platform. With the use of snowball sampling which was used in this study due to the constraints of collecting data during the COVID-19 crisis, a response rate could not be calculated. The survey was kept open for 6 weeks and closed after no responses were received after 2 consecutive weeks.

\section{Data Analysis}

We used an a priori, deductive process to categorise the qualitative data from SWOT analysis. All investigators first read through the data set several times to become familiarized with the data. We used descriptive and in vivo coding to generate the themes that would form the object of analysis. ${ }^{20}$ Hence, the responses to the openended questions were thematically analyzed through inductive, iterative reviews by all three investigators (VSS, KN \& SS).$^{20}$ In thematic analysis the data set is analysed to look for common views and issues that recur to ensure that the main themes identified summarise all the responses collected. ${ }^{18,21}$ To enhance the reliability of the analysis the themes and subthemes were compared and discussed until consensus was reached by all investigators. ${ }^{21}$ A summary of the themes, with supporting quotes, are described in the next section. Trustworthiness of the data was thus enhanced through data organization and ensuring data accuracy, dependability and transferability (use of thick descriptions). Participants were represented by codes, for instance P1, to ensure that the data was reported anonymously.

\section{Results}

\section{Respondents' Demographic Profiles}

Our survey yielded 155 respondents across the country of which $64 \%(\mathrm{n}=99)$ were female, $79 \%$ unmarried $(\mathrm{n}=117)$ and $71 \%(n=104)$ identifying as urban-based. There was a spread of respondents from all the major healthcare SA universities $(77 \%)$ even though $23 \%$ of the respondents chose not to disclose their university affiliation. The majority of the participants were final-year medical students 
(68\%, $\mathrm{n}=100 / 147)$. The remainder were nursing (14/147), physiotherapy (12/147), occupational therapy ( $9 / 147)$, medical orthotics and prosthetics (3/147), dental (4/147), optometry (2/147), clinical associates (2/147) and dietetics (1/ 147) final-year students.

Seventeen major themes related to SDL were identified. As illustrated in Table 1, themes identified within the strengths and opportunities domains are reported as enablers, while themes within the weaknesses and threats domains are reported as barriers to SDL.

\section{Enablers to SDL}

The six themes identified as enablers for SDL development are illustrated with supportive quotes in this section.

\section{Personal Skill Sets}

Students identified various skill sets related to selfdetermination and self-preservation that supported them positively and enabled their ability to take up learning despite the challenges posed by the COVID-19 situation. These related to inherent traits within students.

I believe I cope well with stress and manage to work effectively in stressful situations. COVID has definitely increased the stress related to my final year, but I feel that I am resilient enough to overcome any issues. I am independent can take initiative to self-study etc, whereas other students may require more assistance. (P40, Medicine)

Although the poor socio-economic circumstances of students were described as a learning barrier, they also served as a motivating factor to continue learning so that students

Table I Enablers and Barriers to SDL Themes Within a SWOT Framework

\begin{tabular}{|c|c|}
\hline \multicolumn{2}{|c|}{ Enablers } \\
\hline $\begin{array}{l}\text { Strengths } \\
\text { - Personal skill sets } \\
\text { - Learning behaviours and skills }\end{array}$ & $\begin{array}{l}\text { Opportunities } \\
\text { - Online education } \\
\text { - Collaborative learning networks } \\
\text { - Health advocacy } \\
\text { - Infection control practices }\end{array}$ \\
\hline \multicolumn{2}{|c|}{ Barriers } \\
\hline $\begin{array}{l}\text { Weaknesses } \\
\text { - Fear and anxiety } \\
\text { - Infection of self and others } \\
\text { - Untrusted learning sites } \\
\text { - Uncertainty about graduation } \\
\text { - Financial issues }\end{array}$ & $\begin{array}{l}\text { Threats } \\
\text { - Quarantine } \\
\text { - Poor communication } \\
\text { - Lack of online learning experiences } \\
\text { - Lack of optimal PPE } \\
\text { - Lack of face-to-face training } \\
\text { - Lack of study space and interrupted } \\
\text { connectivity }\end{array}$ \\
\hline
\end{tabular}

could complete their degrees and improve their socioeconomic circumstances.

I want to finish my career so that I will be able to support my family. (P119, Medicine)

Completion of degrees to help combat poverty. (P90, Medicine)

Students were eager to learn new things to enhance their knowledge and development as healthcare professionals.

New ways of thinking, new skills to learn, advocacy of profession in the field, new roles for the profession to fill and implement. (P147, Occupational Therapy)

Committed to learning and adjusting my skills to adjust to the COVID time. (P136, Physiotherapy)

\section{Learning Behaviors and Skills}

Many students identified and embraced certain learning behaviors that supported self-directed learning as a strength and opportunity to take responsibility for their own learning.

I have all the necessary study material plus laptop and cellphone for internet learning. I was fully aware of what was happening and I fully understand that it was necessary. The university prepared us with all the information we need to have as far as Covid is concerned. In sixth year, most of the work is revision so individual studying is not that much of a problem. (P88, Medicine)

Regular academic programs were suspended due to the lockdown. The additional time created encouraged students to become more autonomous and self-disciplined and to create their own learning goals.

The additional time and slower pace as well as reduced expectations means I have been able to cement my theoretical knowledge and improve my clinical reasoning process. (P149, Occupational Therapy)

Studying at my own pace and finding gaps of knowledge. (P9, Nursing)

\section{Online Education}

Online learning platforms and resources motivated selflearning skills and the use of evidence-based practices. The increased exposure to a wider spectrum of both knowledge sources, material, and platforms was viewed positively in stimulating individual learning.

Making use of online learning resources from other developed countries. (P58, Medicine) 
Keep updated with latest news, research, precautions, PPE training, etc. (P100, Medicine)

\section{Collaborative Learning Networks}

Students sought out collaborative learning networks with other learners to facilitate their learning needs and goals.

I continue to collaborate with my [fellow] learners to have some clarity for my courses. (P4, Nursing)

During this pandemic, I tried to work with different groups, collaborating with them how to do the work and protect our lives. (P2, Nursing)

\section{Health Advocacy}

Students described themselves as caring and empathetic towards their patients

Caring, respect and having relevant clinical competences. (P8, Nursing)

I can help in whatever am able to do and trained to do to save lives. (P11, Nursing)

The attributes of care and empathy that students reported were invoked in expressions of health advocacy in which they displayed a vocational sense of duty and responsibility towards others. Students reported on having a desire to educate their families, friends and communities about COVID-19. Some students reported developing a patient rights advocacy approach to their training.

Give awareness to the community, respect people's privacy and dignity, trustworthy and easy to communicate with, be dedicated and disciplined, give hope to the community. (P83, Medicine)

To be able to advocate for a patient in cases where she/he do not understand something or her/his rights are being violated. (P11, Nursing)

\section{Infection Control Practices}

Students also diagnosed the need for improving knowledge, skills, and attitudes related to infection control practices. They acknowledged an increase in these opportunities around the use of PPE and infection control practices. This highlighted the enabling aspect of the pandemic to SDL.

We have the opportunity of learning more about safety in the workplace; how to ensure the safety of ourselves and our patients by using PPEs appropriately (P62, Medicine)

\section{Barriers to SDL}

Ten themes were identified as barriers for the development of SDL in students. These themes are illustrated with supportive quotes in this section.

\section{Fear and Anxiety Related to Infecting Self and Others} A major theme related to barriers to learning was participants' fear of the unknown. This notion of fear was linked to perceptions of compromised personal health due to contracting coronavirus through contact in the clinical environment. Additionally, this fear was not limited to the individual. There were concerns that the infection could be passed to family members and loved ones.

Fear of being a carrier and infecting people. Being in a clinical setting is a high risk, which plays on my mind constantly. Knowing that I have high risk family members at home makes me scared to go back. (P143, Physiotherapy)

This risk was seen as further compounded by the individual or family member's susceptibility to infection due to underlying health comorbidities.

\section{Untrusted Learning Sites}

The fear and anxiety felt were seemingly exacerbated by an apparent lack of support, concern or even trust. Students viewed the move to return final years to workplace learning as a pretext to ensure the throughput that the students to graduation so that the following years workforce is not compromised. An outcome of suspicion in such circumstances might demotivate students. This may lead them to question their learning needs, since it might present a disjuncture between their learning goals and expectations and those of their institutions.

Concern that the university and Department of Health will be exploiting us workhorses to support this strained health system under the pretext of us going back to clinical rotations for learning purposes ... to ensure that there are enough interns next year without considering the implications of returning to health sites too early. (P92, Medicine)

\section{Uncertainty About Graduation}

The uncertainty related to whether or not students would be able to complete the academic year and graduate were strong themes in the student data. With questions about graduation lingering in the air unaddressed, students' learning and goals were disrupted. This created mental anguish and pleas for help. 
We wish a decision can be made and assurance can be given to us on the outlook of finishing our degree, as the anxiety and uncertainty is taking mental tolls on us ... We need concrete ways to move forward and to be told what will happen if we contract the virus and its effect on finishing academic requirements to pass blocks and degree. Please help us. (P100, Medicine)

The following question highlights an added burden of stress brought on by the lack of contingency plans to facilitate learning goals and needs. Without this clarity, concrete decisions around whether students should continue to study or perhaps continue preparing for exams hang in the balance until the question of graduation is resolved. These stresses demotivate learners and negatively impact their commitment to SDL.

As a final year student contracting COVID-19 during this crucial time ... does that mean pausing my academic year until next year or will I be given an opportunity to join again once am cleared safe? (P58, Medicine)

\section{Financial Issues}

Poor socio-economic backgrounds meant that students' families could not provide financial support to students, particularly those who had elderly parents. This compounded the plight of these students and punctuated their feelings of stress about access to learning resources.

Poor family background, low-income household and elderly parents. (P73, Medicine)

The shutting down of campus infrastructures and libraries compounds the difficulties for students who lack adequate resources at home, as they can no longer take up learning independently, compared with students in better financial positions.

Personal financial circumstance and reduced access to campus resources mean less access to essential resources such as books. (P102, Medicine).

\section{Quarantine}

The quarantine imposed on infected patients presented the real possibility of eliminating students from the field of learning. Quarantine entailed isolation from others and being taken away from collaborative learning networks that usually addressed their learning needs (enablers). It also contained the possibility that their learning came to a complete halt due to tight infection control procedures at quarantine sites, which might not have allowed personal learning resources like books and laptops for infected students to continue their learning.

Negative impact on my learning as well as that of peers should I test positive and be required to quarantine. (P92, Medicine)

If one of us is infected, we will all have to be checked and quarantined until we are all cleared, which will take away another precious time of learning and engaging with our studies (P157, Dentistry).

\section{Poor Communication}

The perceived threats to learning included lack of proper communication between clinical tutors, lecturers, and students, high clinical expectations, and disorganization in the learning environment.

Lack of communication from faculty about the next steps, workload being pushed into a smaller time frame makes things far more stressful. (P77, Medicine)

\section{Lack of Online Learning Experiences}

Another barrier was respondents' lack of preparedness and inexperience to deal with the changes in the learning environment.

Poor coping mechanisms, unable to teach myself online without any seniors' assistance. (P20, Medicine)

Distance learning means no first-hand experience. (P129, Occupational Therapy)

\section{Lack of Optimal PPE}

Another theme to emerge was the suboptimal response from the clinical settings to enable and facilitate student learning. From a clinical learning perspective, respondents believed that the lack of PPE was a major barrier to safe patient management. This was further compounded by insufficient equipment and lack of appropriate infrastructure in the clinical setting. The clinical setting was seen as an unsafe environment. Overcrowding in public sector hospital settings meant social distancing was a challenge in the clinical environment.

Lack of PPE and a safe environment for learning and working. (P98, Medicine)

Resources such as gloves are scarce and therefore will prove to be a challenge as we become in physical contact with patients. (P127, Medical Orthotics and Prosthetics) 


\section{Lack of Face-to-Face Training}

Lack of face-to-face training compromised academic support, clinical training, and learning goals related to the development of core competencies.

No tutorials. Only online, cannot ask questions in real time. Ward rounds difficult. (P17, Medicine).

Reduced clinical exposure and interaction with patients, hence less time to improve clinical skills and practice professional core competencies. (P104, Medicine)

This conundrum was further punctuated by inadequate learning platforms, non-availability of clinical teaching staff and limited access to non-clinical training sites. Challenges in accessing clinical teaching staff could inevitably result in a suboptimal clinical learning environment.

Registrars do not want to teach any students and therefore students are left to self-study or sit and do nothing the whole day. (P24, Medicine)

\section{Lack of Study Space and Interrupted Connectivity}

Other themes were linked to inadequate external support for student learning. Study space constraints, quality of internet connectivity for online learning, and interruptions to electricity supply threatened the SDL.

[Interrupted] internet connection and electricity. (P129, Occupational Therapy)

Lack of personal space and environment to study optimally ... quality of lecture carried out on online platforms highly dependent on internet connection quality. (P102, Medicine)

\section{Discussion}

This study identified various enablers and barriers to SDL among final-year students across various health professions disciplines. These could be viewed as internal factors inherent to students over which they seemed to have control and external environmental factors, which students seemed powerless to influence.

Integral to an SDL approach is an individual reflection in diagnosing one's learning needs. ${ }^{11}$ However, in this study, these needs seemed camouflaged in expressions of fear and anxiety concerning contracting COVID-19, spreading it to their loved ones, and not being able to graduate timeously. Islam et al reported that more than two-thirds of students surveyed in their study experienced mild to severe depression (82.4\%) and anxiety (87.7\%), especially linked to concerns of transmitting the infection to family members and loved ones. ${ }^{22}$ These findings are consistent with the assertions of Sahu that, given the interruptions in teaching and learning due to COVID-19, students may graduate later than usual. ${ }^{23}$ This could affect graduates negatively, especially students from disadvantaged socio-economic settings, as their priority would be to start working as soon as possible.

The challenging learning environment, as reflected by many students due to suboptimal allocation of PPE and the sudden lack of face-to-face learning, created feelings of powerlessness for medical students. This feeling of powerlessness posed a challenge seen through the SDL lens, where choosing and implementing appropriate learning strategies and formulating goals should be a cornerstone. ${ }^{13,16}$ These perceptions among students possibly reflect a lack of preparedness to deal with the changes in the learning environment and could contribute to a lack of confidence in SDL. This study's findings are consistent with those of Choi et al, who reported that $59.3 \%$ of the students in their study felt less prepared while $22.7 \%$ felt less confident. ${ }^{24}$ This lack of preparedness to adopt new and more appropriate strategies was also noted by Rafi et al. ${ }^{6}$ Here, the authors found that only a few students in their study supported the notion of continuing online classes once the regular classroom-based teaching was reinstated. Students still wanted a repetition of the classes when the normal academic program resumed. This suggests that the value placed on face-to-face classroom and clinical setting environments should not be ignored. Similarly, Hammond et al reported that online teaching and learning cannot substitute real-world patient contact and time in the clinical environment. ${ }^{25}$ Rajab et al, on the other hand, reported that most participants in their study preferred a combination of online and face-to-face learning. ${ }^{3}$ These findings will influence institutions and teachers in considering a hybridized approach to health professions education, especially. ${ }^{26}$

Essential elements to ensure self-directedness in learning include the role of the educator as a facilitator and not a content source, students having some degree of agency over the learning resources, and being involved in selfassessment with learning contracts. ${ }^{11,13}$ If we cultivate these activities in students, the final years of most health professions practice provide ample opportunities. Various skill sets were self-identified by students, providing coping strategies and ensuring resilience during the COVID-19 pandemic. Other skill sets identified included the need for health professions students to be advocates for patients and to be active participants in collaborative teams. 
Similar to other reports, this study affirms that, although SDL implies individual effort, it may include a collaborative process. ${ }^{14} \mathrm{We}$ found that closing residences, libraries, and learning spaces disrupted the collaborative learning practices of some students, which negatively influenced their learning initiatives. However, students used online platforms and found other creative ways to reignite their study circles, facilitating their learning goals.

Further, our study supports that SDL can be used as a tool during uncertain times. ${ }^{14}$ During the COVID-19 crisis, we found that students used the disrupted learning timetables to become autonomous and self-disciplined and created their own learning goals based on their individual learning needs. Access to newly created online learning platforms and global resources fueled their desire to learn new things, specifically more about their professions and the new COVID-19 disease processes. Hence, the abrupt changes brought on by the challenges of a global pandemic challenged students to accept responsibility for their own learning. This study found that, while students recognized their weaknesses, they were motivated by a desire to graduate and become competent healthcare professionals. Using their strengths and resilience, they capitalized opportunities to "maximise their learning in the new normal". ${ }^{16}$

As the future custodians of public health emergencies, final-year health professions students cannot be considered as non-essential in clinical delivery. ${ }^{6}$ These students would be a valuable resource to the health workforce, but this should be carefully supplemented with clear guidance for students to ensure that such clinical exposure directly impacts their required competencies. There should also be "pastoral support, and appropriate remuneration" for the students' time. ${ }^{21}$ The issue of remuneration for final-year students in the clinical setting has been previously noted and was practiced with stipends. Still, this learning contract of "junior internship" needs relooking at. It requires a broader dialogue with all stakeholders involved in clinical training. There is a need for inclusive planning, decision-making, and recognizing the duality in student roles and responsibilities during a pandemic. This public health emergency has highlighted the need to revisit student clinical training.

\section{Strengths and Limitations of the Study}

This study provided valuable insights into students' perspectives on clinical training during the current pandemic; however, some limitations were noted. More research is required on academics' perspectives on clinical teaching during this period. Using social media to recruit study participants could have been a barrier for students with limited or no access to such platforms. However, universities provided access to online data packages. Respondent accountability and veracity in online surveys could affect the quality of responses received. Sampling bias could occur with the snowballing technique; however, the researchers sent the online invitational link to several university training sites so as to attract a wider range of study participants. Generalisability to a national scale is limited as only 155 final-year respondents across several disciplines participated in this study. Additionally, selfreporting could have introduced some bias into the study results, as respondents may have provided responses that they considered to be socially acceptable. Respondents could also have had challenges in assessing themselves accurately. Nonetheless, this study provides a snapshot of SDL during the COVID-19 pandemic. Further, we note that survey-based studies may limit an in-depth analysis. Hence, we recommend that future studies include focus groups and interviews to gain a much more in-depth analysis of SDL experiences of clinical training during a crisis.

\section{Conclusion}

The impact of the COVID-19 pandemic on final-year health professions students, viewed through the lens of SDL, provides a context for reflection and review. Engagement with final-year students to address identified stressors helps support the call for health professional educators to prepare self-directed learners. This study identifies the COVID-19 pandemic as a health crisis that acted as an environmental barrier to developing effective SDL skills. Responsive curricula that optimize unpredictable disruptions in clinical training are needed to equip students to diagnose their own learning needs and implement appropriate learning strategies. The use of hybridized training programmes that encompass online and face-toface clinical teaching in the final year of training permits greater clinical exposure and is seen as an enabling learning strategy. Providing a safe and supportive workplace as part of the educators' responsibilities is viewed as a mitigation against these challenges in the environment. Shared platforms where students are part of the decisionmaking processes for clinical learning and training are thus recommended if SDL skills are to be optimised during crises. Final-year health professions students should enter 
into "learning contracts" that see them as "junior interns" with possible remunerative stipends. This could see them as integral and of assistance in health crises, specifically enabling SDL and assessment activity before graduation.

\section{Data Sharing Statement}

The data sets used and analyzed during the current study are available from the corresponding author on reasonable request.

\section{Ethics Approval and Consent to Participate}

This study received approval from the University of KwaZulu-Natal Ethics Committee (HSSREC/00001306/ 2020). All participants provided informed consent.

\section{Disclaimer}

The views expressed in the submitted article are the authors' own and not an official position of the institution.

\section{Acknowledgments}

Special thanks to all the final-year medical students who made time to respond and share the questionnaire with their colleagues.

\section{Author Contributions}

All authors made a significant contribution to the work reported. This relates to the study design, execution, acquisition of data, analysis and interpretation. All the authors took part in drafting, revising or critically reviewing the article; gave final approval of the version to be published; have agreed on the journal to which the article has been submitted; and agree to be accountable for all aspects of the work.

\section{Disclosure}

The authors declare that they have no competing interests in this work.

\section{References}

1. Singh S, Roy D, Sinha K, Parveen S, Sharma G, Joshi G. Impact of COVID-19 and lockdown on mental health of children and adolescents: a narrative review with recommendations. Psychiatry Res. 2020;293:113429. doi:10.1016/j.psychres.2020.113429

2. Al-Rabiaahab A, Temsah MH, Al-Eyadhy AA, et al. Middle East Respiratory Syndrome - corona Virus (MERS-CoV) associated stress among medical students at a university teaching hospital in Saudi Arabia. J Infect Public Health. 2020;13(5):687-691. doi:10.1016/j. jiph.2020.01.005
3. Rajab MH, Gazal AM, Alkattan K. Challenges to online medical education during the COVID-19 pandemic. Cureus. 2020;12(7): e8966. doi:10.7759/cureus. 8966

4. Barton DC. Impacts of the COVID-19 pandemic on field instruction and remote teaching alternatives: results from a survey of instructors. Ecol Evol. 2020;10(22):12499-12507. doi:10.1002/ ece3.6628

5. Sharma S, Sharma V. Medical education during the COVID-19 pandemics - challenges ahead. Indian Pediatr. 2020;57(8):772. doi:10.1007/s13312-020-1940-5

6. Rafi AM, Varghese PR, Kuttichira P. The pedagogical shift during COVID 19 pandemic: online medical education, barriers and perceptions in central Kerala. J Med Educ Curr Dev. 2020;7:1-4. doi: $10.1177 / 2382120520951795$

7. Timmis S, Broadfoot P, Sutherland R, Oldfield A. Rethinking assessment in a digital age: opportunities, challenges and risks. Br Educ Res J. 2015;42(3):454-476. doi:10.1002/berj.3215

8. Gill D, Whitehead C, Wondimagegn D. Challenges to medical education at a time of physical distancing. Lancet. 2020;396 (10244):77-79. doi:10.1016/S0140-6736(20)31368-4

9. Ferrel MN, Ryan JJ. The impact of COVID-19 on medical education. Cureus. 2020;12(3):e7492. doi:10.7759/cureus. 7492

10. American Board of Internal Medicine: Maintenance of Certification. Accreditation Council for Graduate Medical Education. ACGME Outcome Project. Philadelphia (PA): ABIM; 2007.

11. Li ST, Paterniti DA, Co JP, West DC. Successful self-directed lifelong learning in medicine: a conceptual model derived from qualitative analysis of a national survey of pediatric residents. Acad Med. 2010;85(7):1229-1236. doi:10.1097/ACM.0b013e3181e1931c

12. Simon FA, Aschenbrener CA. Undergraduate medical education accreditation as a driver of lifelong learning. J Contin Educ Health Prof. 2005;25(3):157-161. doi:10.1002/chp.23

13. Knowles M. Self-Directed Learning: A Guide for Learners and Teachers. New York (NY): Associated Press; 1975.

14. Roberson DN Jr, Zach S, Choresh N, Rosenthal I. Self-directed learning: a longstanding tool for uncertain times. Creat Educ. 2021;12:1011-1026. doi:10.4236/ce.2021.125074

15. van Woezik T, Koksma J, Reuzel R, Jaarsma DC, van der Wilt GJ. There is more than ' $I$ ' in self-directed learning: an exploration of selfdirected learning in teams of undergraduate students. Med Teach. 2021;43(5):590-598. doi:10.1080/0142159X.2021

16. Mahlaba SC. Reasons why self-directed learning is important in South African during the COVID-19 pandemic. SAJHE. 2020;34 (6):120-136. doi:10.20853/34-6-4192

17. Emet G, Tat M. Swot analysis: a theoretical review. J Int Soc Res. 2017;10(51):994-1006. doi:10.17719/jisr.2017.1832)

18. Braun V, Clarke V, Boulton E, Davey L, McEvoy C. The online survey as a qualitative research tool. Int J Soc Res Methodology. 2020,16,1-4

19. van Wijngaarden JD, Scholten GR, van Wijk KP. Strategic analysis for health care organizations: the suitability of the SWOT-analysis. Int J Health Plann Manage. 2012;27(1):34-49. doi:10.1002/hpm.1032

20. Saldaña J. The Coding Manual for Qualitative Researchers. 3rd ed. Los Angeles (CA): SAGE; 2015.

21. Varpio L, Ajjawi R, Monrouxe LV, O’Brien BC, Rees CE. Shedding the cobra effect: problematising thematic emergence, triangulation, saturation and member checking. Med Educ. 2017;51(1):40-50. doi:10.1111/medu.13124

22. Islam MA, Barna SD, Raihan H, Khan MNA, Hossain MT. Depression and anxiety among university students during the COVID-19 pandemic in Bangladesh: a web-based cross-sectional survey. PLoS One. 2020;15(8):e0238162. doi:10.1371/journal. pone. 0238162

23. Sahu P. Closure of universities due to coronavirus disease 2019 (COVID-19): impact on education and mental health of students and academic staff. Cureus. 2020;12(4):e7541. doi:10.7759/ cureus. 7541 
24. Choi B, Jegatheeswaran L, Minocha A, Alhilani M, Nakhoul M, Mutengesa E. The impact of the COVID-19 pandemic on final year medical students in the United Kingdom: a national survey. $B M C$ Med Educ. 2020;20(1):206. doi:10.21203/rs.3.rs-24792/v1

25. Hammond D, Louca C, Leeves L, Rampes S. Undergraduate medical education and Covid-19: engaged but abstract. Med Educ Online. 2020;25(1):1781379. doi:10.1080/10872981.2020.1781379
26. Comas-Quinn A. Learning to teach online or learning to become an online teacher: an exploration of teachers' experiences in a blended learning course. ReCALL. 2011;23(3):218-232. doi:10.1017/ S0958344011000152

\section{Publish your work in this journal}

Advances in Medical Education and Practice is an international, peerreviewed, open access journal that aims to present and publish research on Medical Education covering medical, dental, nursing and allied health care professional education. The journal covers undergraduate education, postgraduate training and continuing medical education including emerging trends and innovative models linking education, research, and health care services. The manuscript management system is completely online and includes a very quick and fair peer-review system. Visit http://www.dovepress.com/testimonials.php to read real quotes from published authors. 